\title{
Impact and mechanism of non-steroidal anti-inflammatory drugs combined with chemotherapeutic drugs on human lung cancer-nude mouse transplanted tumors
}

\author{
WEIYI SUN ${ }^{1}$ and GANG CHEN ${ }^{2}$ \\ Departments of ${ }^{1}$ Emergency and ${ }^{2}$ Respiratory, The Third Hospital of Hebei Medical University, \\ Shijiazhuang, Hebei 050051, P.R. China
}

Received November 26, 2014; Accepted December 14, 2015

DOI: $10.3892 / 01.2016 .4493$

\begin{abstract}
The present study aimed to investigate the impact of indomethacin treatment combined with oxaliplatin treatment on the expression of cluster of differentiation 44 variant 6 (CD44v6), matrix metalloproteinase-2 (MMP-2) and survivin in human lung cancer-nude mouse transplanted tumors. The human lung adenocarcinoma (A549)-nude mouse transplanted tumor model was established, and the mice were divided into a control group, an indomethacin treatment group, an oxaliplatin treatment group and an indomethacin-oxaliplatin combination treatment group. The tumor inhibition rate was calculated following sacrificing of the mice. Immunohistochemical staining and fluorescence reverse transcription-quantitative polymerase chain reaction were utilized to detect the protein and messenger (m)RNA expression of CD44v6, MMP-2 and survivin. The tumor inhibition rates of the indomethacin group, the oxaliplatin group and the combination group were $26.67,47.70$ and $68.88 \%$, respectively. The protein and mRNA expression levels of CD44v6, MMP-2 and survivin in the transplanted tumors of each treatment group were reduced compared with the control group $(\mathrm{P}<0.05)$, and those of the combination group were lower compared with the single-drug treatment groups $(\mathrm{P}<0.05)$. Survivin and MMP-2, MMP-2 and CD44v6, and MMP-2 and CD44v6 all exhibited linear positive correlation. The present study provides evidence that the administration of indomethacin alone, or in combination with oxaliplatin, may significantly inhibit the growth of lung cancer-nude mouse transplanted tumors and the expression of CD44v6, MMP-2 and survivin inside the tumor. The combination of non-steroidal anti-inflammatory drugs with chemotherapeutic drugs may improve the antitumor effects.
\end{abstract}

Correspondence to: Dr Gang Chen, Department of Respiratory, The Third Hospital of Hebei Medical University, 139 Ziqiang Road, Shijiazhuang, Hebei 050051, P.R. China

E-mail: gangchendoc@126.com

Key words: lung cancer, non-steroidal anti-inflammatory drugs, chemotherapy, cluster of differentiation 44 variant 6 , matrix metalloproteinase-2, survivin

\section{Introduction}

The invasion and metastasis of lung cancer is a complex process that is regulated by multiple genes, and the interaction between these genes has become a significant research focus. Cyclooxygenase-2 (COX-2) is the key enzyme involved in the occurrence and development of a variety of malignant tumors $(1,2)$. COX-2 expression promotes proliferation, angiogenesis and lymphangiogenesis within tumor cells, as well as promoting tumor invasion and metastasis (3-5). Cluster of differentiation 44 variant $6(\mathrm{CD} 44 \mathrm{v} 6)$ is a splice variant of CD44, and its expression may alter the composition and functioning of adhesion molecules on the tumor cell surface, thereby contributing to the development of metastatic potential in tumor cells $(6,7)$. Matrix metalloproteinase-2 (MMP-2) is the most widely distributed member of the MMP family, and is able to degrade the extracellular matrix (ECM) and basement membrane, thus participating in physiological and pathological processes including tumor progression $(8,9)$. Survivin is an important member of the inhibitors of apoptosis proteins gene family, and is closely associated with the differentiation, proliferation, angiogenesis, invasion and metastasis of tumor cells $(10,11)$. In the present study, indomethacin, a cyclooxygenase inhibitor, was used in combination with oxaliplatin, a chemotherapeutic drug, to perform intervention treatment on lung cancer-nude mouse transplanted tumors. The present study aimed to research the association between the factors involved in the invasion and metastasis of lung cancer, as well as the mechanism underlying the prevention of tumor growth and metastasis caused by indomethacin and oxaliplatin, thus seeking novel methods to assist with the prevention of lung cancer.

\section{Materials and methods}

Cell culture. Ham's F-12K (Kaighn's) medium (HyClone; GE Healthcare Life Sciences, Logan, UT, USA), containing 10\% fetal bovine serum (FBS; HyClone; GE Healthcare Life Sciences), $100 \mathrm{U} / \mathrm{ml}$ penicillin (North China Pharmaceutical Group Corp., Shijiazhuang, China) and $100 \mathrm{U} / \mathrm{ml}$ streptomycin (Shenzhen HuaYao South Pharmaceutical Co., Ltd., Shijiazhuang, China), was used to subculture A549 human lung 
cancer cells (gifted by Beijing University, Beijing, China) at $37^{\circ} \mathrm{C}$ in an atmosphere of $5 \% \mathrm{CO}_{2}$ and saturated humidity. Cells were used when they were in the logarithmic phase of growth. When microscopy revealed $>80 \%$ of cells demonstrated adherent growth, $0.25 \%$ trypsin (HyClone; GE Healthcare Life Sciences) was used to digest and form the single cell suspension.

Preparation of human lung cancer-nude mouse transplanted tumor model. A total of 32 female BALB/c nude mice, at 4-5 weeks old and weight 20-24 g (obtained from the Institute of Laboratory Animal Science, Chinese Academy of Medical Sciences \& Peking Union Medical College Hospital, Beijing, China; License no. of animal quality: SCXY Jing-2009-0003), were fed with sterile feed and sterile purified water and were housed in individually ventilated cages ( 8 mice per cage). The feeding environment was specific pathogen-free, and the ambient temperature was $25 \pm 2{ }^{\circ} \mathrm{C}$, with a humidity of $45-50 \%$. A549 cells in the logarithmic growth phase were selected, digested and diluted with FBS-free Ham's F-12K (Kaighn's) medium. Subsequently, a hemocytometer was used to adjust the cell density to $1 \times 10^{7}$ cells $/ \mathrm{ml}$. The skin of the nude mouse was disinfected, and $0.2 \mathrm{ml}$ cell suspension was injected with a $1 \mathrm{ml}$ syringe subcutaneously into the left armpit of the nude mouse, in order to establish the lung cancer-nude mouse subcutaneous transplantation model.

Grouping and drug intervention. A total of 32 female BALB/c nude mice were randomly divided into four groups, with 8 mice per group. The groups were as follows: i) indomethacin (Sigma-Aldrich, St. Louis, MO, USA) group, orally administered $2.5 \mathrm{mg} / \mathrm{kg} / \mathrm{day}$, combined with intraperitoneal injection of saline; ii) oxaliplatin (Shandong Lukang Record Pharmaceutical Co., Ltd., Jining, China) group, $10 \mathrm{mg} / \mathrm{kg}$ twice per week, administered by intraperitoneal injection, combined with oral administration of sterile distilled water; iii) indomethacin-oxaliplatin combination group, orally administered indomethacin $2.5 \mathrm{mg} / \mathrm{kg} /$ day, oxaliplatin $10 \mathrm{mg} / \mathrm{kg}$ twice per week, administered by intraperitoneal injection; iv) control group, intraperitoneally injected identical quantity of saline, as well as orally administered sterile distilled water.

Calculation of tumor inhibition rate. The growth of nude mice and tumors were observed, and a caliper was used to measure the long diameter (a) and short diameter (b) of the tumor every 7 days. The formula $\mathrm{V}=\mathrm{ab}^{2} / 2$ was used to estimate the approximate tumor volume. The following formula was used to calculate the percentage tumor inhibition rate $=[1-($ the average volume of each treatment group prior to treatment - the average volume of each treatment group at the conclusion of treatment)/(the average volume of the control group prior to treatment - the average volume of the control group at the conclusion of treatment)] x 100.

Detection of CD44v6, MMP-2 and survivin protein expression by immunohistochemical assay. The primary antibodies survivin rabbit anti-human monoclonal antibody (dilution, 1:100; catalog no., ZA-0530), CD44v6 mouse anti-human monoclonal antibody (dilution, 1:300; catalog no., ZM-0052) and MMP-2 mouse anti-human monoclonal antibody (dilution,
1:300; catalog no., ZM-0330) and the monoclonal mouse anti-human IgG secondary antibody (dilution, 1:100; catalog no., PV6002) were purchased from Beijing Zhongshan Golden Bridge Biotechnology Co., Ltd. (Beijing, China). Immunohistochemistry parvalbumin kit was purchased from OriGene Technologies (Beijing, China). Paraffin sections were conventionally deparaffinized, followed by high pressure antigen repairing. Subsequently, the sections were incubated with primary and secondary antibodies, followed by 3,3'-diaminobenzidine staining (Beijing Zhongshan Golden Bridge Biotechnology Co., Ltd.), hematoxylin (Beijing Zhongshan Golden Bridge Biotechnology Co., Ltd.) restaining, differentiation, blue-restoring, dehydration and mounting. Subsequently, the sections were observed and images were captured under an optical microscope (BX51; Olympus Corporation, Tokyo, Japan). The CD44v6 positive indicator was brown staining, located in the cell membrane and/or cytoplasm. The MMP-2 positive indicator was brown staining, located in the cell membrane and/or cytoplasm. The survivin positive indicator was brown staining, located in the nucleus and/or cytoplasm. A general observation of the tissue sections was initially performed using a microscope, under low magnification, and subsequently 10 high-power fields were randomly selected for observation under magnification, x200. The Beihang true color pathological image analysis system (Beihang University, Beijing, China) was used to calculate average integrated absorbance values.

Detection of CD44v6, MMP-2 and survivin mRNA expression by fluorescence reverse transcription-quantitative polymerase chain reaction $(R T-q P C R)$ assay. TRIzol ${ }^{\circledR}$ reagent (RNA extraction solution) was purchased from Invitrogen (Thermo Fisher Scientific, Inc., Waltham, MA, USA). Reverse transcriptase, RNasin ${ }^{\circledR}$ inhibitor, deoxynucleotides, TaqDNA polymerization enzyme and agarose were purchased from Promega Corp. (Madison, WI, USA). Random primers were synthesized by Sangon Biotech Co., Ltd (Shanghai. China). RealMasterMix (SYBR Green) kit and DNAse was purchased from Tiangen Biotech Co., Ltd. (Beijing, China). The tumor tissue RNA was extracted using $2 \mu \mathrm{l}$ DNAse per sample, and a UV spectrophotometer (UV-2550 2450; Shimadzu, Corp., Kyoto, Japan) was used to detect the optical density (OD) 260/OD280 ratio. If this ratio was 1.8-2.0, it indicated that the extracted RNA was slightly contaminated. Subsequently, reverse transcription was performed to produce cDNA. The primers used were as follows: Glyceraldehyde-3-phosphate dehydrogenase (GAPDH) forward, 5'-TGAACGGGAAGC TCACTGG-3' and reverse, 5'-GCTTCACCACCTTCTTGA TGTC-3'; CD44v6 forward, 5'-GGAGCCAAATGAAGA AAATGAA-3' and reverse, 5'-TGAAATGGTGCTGGAGAT AAAA-3'; MMP-2 forward, 5'-AACTACGATGATGACCGC AAG-3' and reverse, 5'-GACAGACGGAAGTTCTTGGTG-3'; survivin forward, 5'-TTTCTCAAGGACCACCGCA-3' and reverse, 5'-AGTCTGGCTCGTTCTCAGTG-3'. The total PCR reaction volume was $20 \mu 1$, and the PCR thermal cycling parameters were as follows: $95^{\circ} \mathrm{C}$ for $2 \mathrm{~min}$, followed by $95^{\circ} \mathrm{C}$ for $15 \mathrm{sec}, 59^{\circ} \mathrm{C}$ for $30 \mathrm{sec}$ and $68^{\circ} \mathrm{C}$ for $40 \mathrm{sec}$ for 40 cycles. An ABI 7500 Real-Time PCR System (Thermo Fisher Scientific Inc.) was used. The fluorescence signal was collected at the third step $\left(59^{\circ} \mathrm{C}\right.$ for $\left.30 \mathrm{sec}\right)$ of each cycle. The experiment 
was performed 5 times. Following amplification, the results were analyzed, and the target gene expression levels were normalized against GAPDH, thus the relative quantification value (RQ value) of the target gene expression was obtained. The RQ values were subsequently used for statistical analysis, as described previously (12).

Statistical analysis. All obtained experimental data were statistically analyzed with SPSS version 17.0 (SPSS, Inc., Chicago, IL, USA). The data of each group were expressed as the mean \pm standard deviation. Intergroup comparison was performed using analysis of variance when the data displayed homogeneity of variance, and if it did not a Kruskal-Wallis rank-sum test was performed. $\mathrm{P}<0.05$ was considered to indicate a statistically significant difference. The correlation among the factors was analyzed using the Pearson product-moment correlation coefficient, with $\mathrm{P}<0.01$ considered to indicate a statistically significant difference.

\section{Results}

Drug treatment reduces the growth of human lung cancer-nude mouse transplanted tumors. The 32 mice were all tumorigenic, with a tumor formation rate of $100 \%$. A total of 10 days subsequent to transplantation, the average tumor diameter was $4 \mathrm{~mm}$. Following successful tumorigenesis, the early weight and activities of nude mice did not change significantly; however, following tumor growth, the nude mice exhibited weight loss and gradual decreased activity. No mouse succumbed to disease during the experimental period (Fig. 1).

Following treatment, the transplanted tumor volume of the control group was 1,643.12 $\pm 204.65 \mathrm{~mm}^{3}$, while those of the indomethacin group, the oxaliplatin group and the combination group were $1,450.29 \pm 133.20 \mathrm{~mm}^{3}$, $743.84 \pm 151.55 \mathrm{~mm}^{3}$ and $568.69 \pm 119.58 \mathrm{~mm}^{3}$, respectively. Tumor volume comparison amongst the experimental groups revealed a statistically significant difference $(\mathrm{F}=75.697 ; \mathrm{P}<0.01)$. The tumor inhibition rates of the indomethacin group, the oxaliplatin group and the combination group were $26.67,47.70$ and $68.88 \%$, respectively, and the tumor inhibition rate of the combination group was significantly increased compared with the monotherapy groups $(\mathrm{P}<0.05)$.

Drug treatment reduces the protein expression of $C D 44 v 6$ $M M P-2$ and survivin. The protein expression levels of CD44v6, MMP-2 and survivin in the indomethacin group, the oxaliplatin group and the combination group were reduced compared with the control group $(\mathrm{P}<0.05)$, and the levels in the combination group were reduced compared with the monotherapy groups $(\mathrm{P}<0.05)$. However, the expression of CD44v6, MMP-2 and survivin proteins between the indomethacin group and the oxaliplatin group did not exhibit a significant difference (P>0.05; Table I; Figs. 2-4)

Drug treatment reduces the $m R N A$ expression of $C D 44 v 6$ $M M P-2$ and survivin. The mRNA expression levels of CD44v6, MMP-2 and survivin in the indomethacin group, the oxaliplatin group and the combination group were reduced compared with the control group $(\mathrm{P}<0.05)$, and the levels in the combination group were reduced compared with the monotherapy group

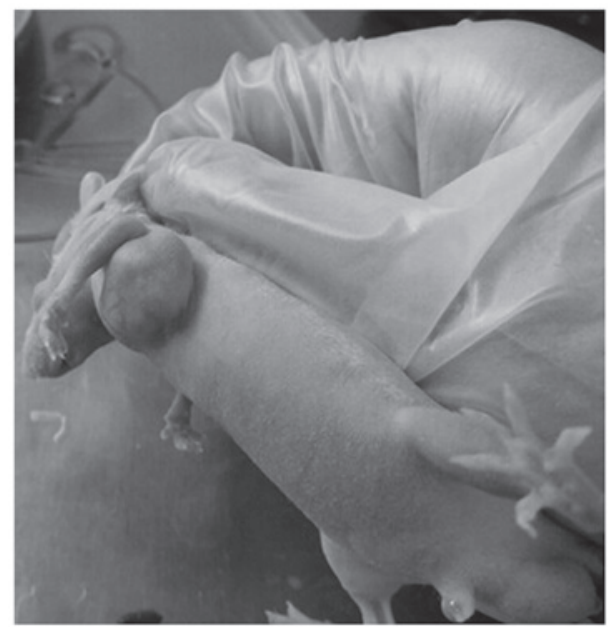

Figure 1. Model of human lung cancer-nude mouse transplanted tumors.

$(\mathrm{P}<0.05)$. However, the expression of CD44v6, MMP-2 and survivin mRNAs between the indomethacin group and the oxaliplatin group did not exhibit a significant difference (P>0.05; Table II).

Correlation analysis results. In the combination group, it was found that MMP-2 protein and MMP-2 mRNA expression were positively correlated $(\mathrm{r}=0.958 ; \mathrm{P}<0.01)$. CD44v6 protein and CD44v6 mRNA expression were positively correlated ( $r=0.906$; $\mathrm{P}<0.01)$. Survivin protein and survivin mRNA expression were positively correlated $(\mathrm{r}=0.931 ; \mathrm{P}<0.01)$. MMP-2 protein and CD44v6 protein expression were positively correlated $(r=0.907$; $\mathrm{P}<0.01$ ). Survivin protein and MMP-2 protein expression were positively correlated $(\mathrm{r}=0.841 ; \mathrm{P}<0.01)$. Survivin protein and $\mathrm{CD} 44 \mathrm{v} 6$ protein expression were positively correlated $(\mathrm{r}=0.857$; $\mathrm{P}<0.01)$.

\section{Discussion}

Among various types of lung cancer, the expression intensity of COX-2 has been observed to be highest in non-small cell lung cancer, followed by squamous cell carcinoma, large cell lung cancer and certain cases of small cell lung cancer (13). Previous studies have identified that routine administration of nonsteroidal anti-inflammatory drugs (NSAIDs) may reduce the risk of occurrence of colon, breast, lung, prostate and other types of cancer (14). Treatment with the COX-2 inhibitor indomethacin exerts antitumor effects, as well as chemotherapy sensitization, and may regulate the body's immune status (15). Oxaliplatin, as a third-generation anticancer drug, inhibits the synthesis and replication of DNA, thereby preventing the development of tumor cells, and it has been observed to be effective for the treatment of tumors that were resistant to cisplatin (16). Therefore, studying the impact of NSAIDs combined with chemotherapeutic drugs on the tumor growth of a lung cancer-nude mouse transplanted tumor model, as well as the expression of survivin, CD44v6 and MMP-2, may provide a novel method for the treatment and prevention of lung cancer.

The results of the present study demonstrated that the tumor volumes of the drug-treated groups were reduced compared with the control group. The tumor inhibition rates of the 
Table I. Comparison of integral absorbance values of CD44v6, MMP-2 and survivin proteins among the experimental groups (n=8).

\begin{tabular}{lccc}
\hline Variable & CD44v6 & MMP-2 & Survivin \\
\hline Control & $9080 \pm 698$ & $16963 \pm 698$ & $17754 \pm 2505$ \\
Oxaliplatin & $7737 \pm 477^{\mathrm{a}, \mathrm{b}}$ & $14225 \pm 926^{\mathrm{a}, \mathrm{b}}$ & $11945 \pm 1646^{\mathrm{a}, \mathrm{b}}$ \\
Indomethacin & $7706 \pm 504^{\mathrm{a}, \mathrm{b}}$ & $14398 \pm 670^{\mathrm{a}, \mathrm{b}}$ & $12768 \pm 1478^{\mathrm{a}, \mathrm{b}}$ \\
Combination & $5227 \pm 1497^{\mathrm{a}}$ & $9941 \pm 231^{\mathrm{a}}$ & $7317 \pm 1009^{\mathrm{a}}$ \\
P-value & $<0.01$ & $<0.01$ & $<0.01$ \\
\hline
\end{tabular}

${ }^{\mathrm{a}} \mathrm{P}<0.05$ vs. control group; ${ }^{\mathrm{b}} \mathrm{P}<0.05$ vs. indomethacin combined with oxaliplatin-treated group. $\mathrm{CD} 44 \mathrm{v} 6$, cluster of differentiation 44 variant 6 ; MMP-2, matrix metalloproteinase-2; SD, standard deviation. Data presented as the mean \pm standard deviation.

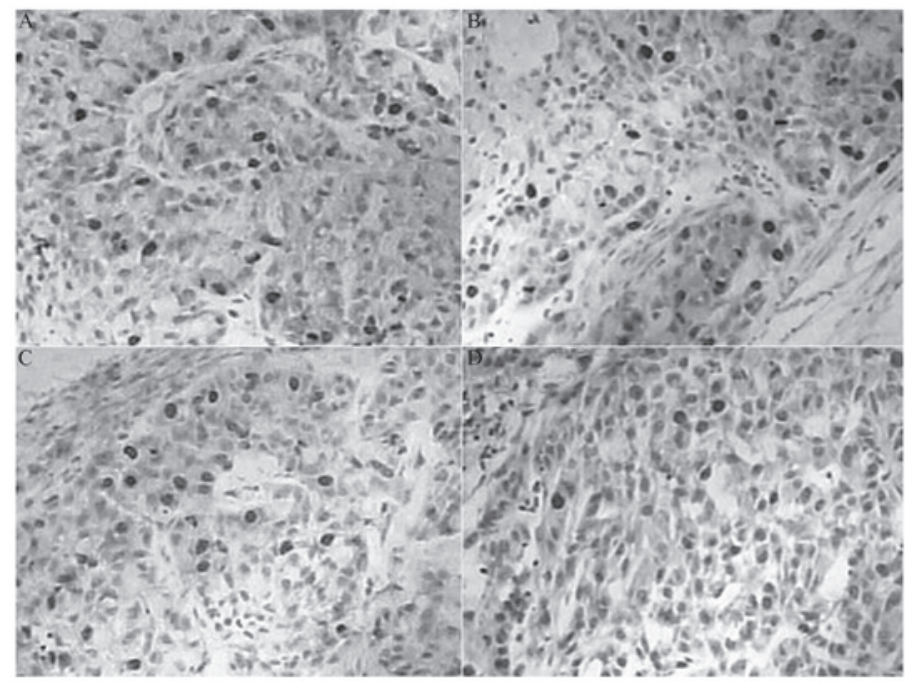

Figure 2. Expression of survivin protein in each group visualized by immunohistochemistry. (A) Control group, (B) indomethacin group, (C) oxaliplatin group and (D) combination group. Magnification, x200.

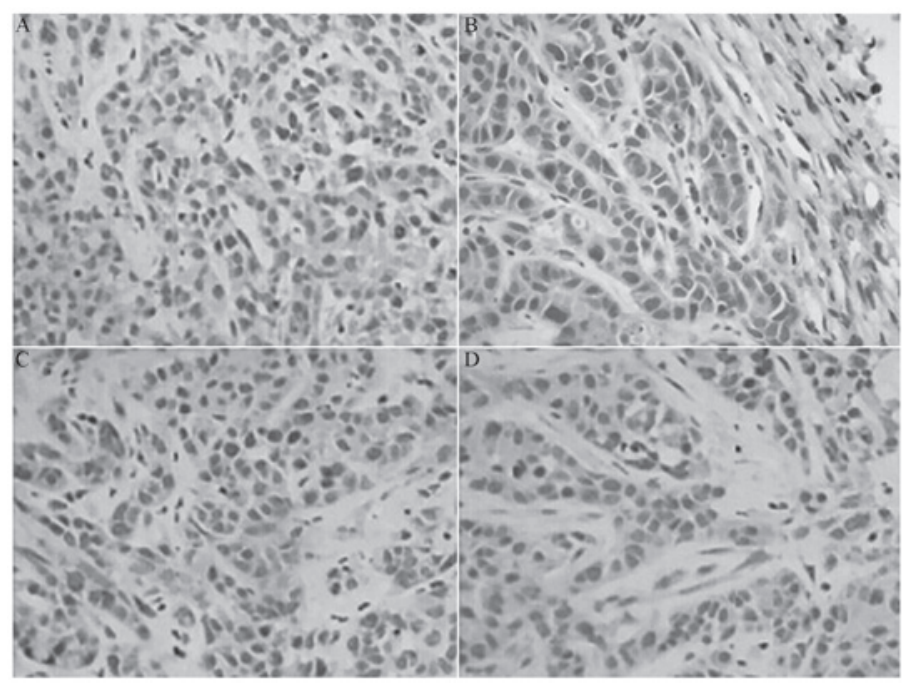

Figure 3. Expression of cluster of differentiation 44 variant 6 protein in each group visualized by immunohistochemistry. (A) Control group, (B) indomethacin group, (C) oxaliplatin group and (D) combination group. Magnification, x200.

indomethacin group and the oxaliplatin group were 26.67 and $47.70 \%$, respectively, while that of the combination group was $71.14 \%$, which was significantly increased compared with the monotherapy groups. This indicated that the combination of
NSAIDs and chemotherapeutic drugs may provide significant synergy for the inhibition of tumor grow th in a lung cancer-nude mouse model. Furthermore, indomethacin has few side effects, its clinical application is wide and it is additionally easy to 
Table II. Comparison of relative quantitative expression values of CD44v6, MMP-2 and survivin mRNAs among the experimental groups $(\mathrm{n}=8)$.

\begin{tabular}{lccc}
\hline Variable & Survivin mRNA & CD44v6 mRNA & MMP-2 mRNA \\
\hline Control & $1.073 \pm 0.078$ & $1.026 \pm 0.037$ & $1.008 \pm 0.017$ \\
Oxaliplatin & $0.617 \pm 0.017^{\mathrm{a}, \mathrm{b}}$ & $0.697 \pm 0.011^{\mathrm{a}, \mathrm{b}}$ & $0.771 \pm 0.012^{\mathrm{a}, \mathrm{b}}$ \\
Indomethacin & $0.616 \pm 0.018^{\mathrm{a}, \mathrm{b}}$ & $0.707 \pm 0.010^{\mathrm{a}, \mathrm{b}}$ & $0.783 \pm 0.011^{\mathrm{a}, \mathrm{b}}$ \\
Combination & $0.426 \pm 0.024^{\mathrm{a}}$ & $0.469 \pm 0.012^{\mathrm{a}}$ & $0.520 \pm 0.011^{\mathrm{a}}$ \\
P-value & $<0.01$ & $<0.01$ & $<0.01$ \\
\hline
\end{tabular}

${ }^{\mathrm{a}} \mathrm{P}<0.05$ vs. control group; ${ }^{\mathrm{b}} \mathrm{P}<0.05$ vs. indomethacin combined with oxaliplatin-treated group. CD44v6, cluster of differentiation 44 variant 6 ; MMP-2, matrix metalloproteinase-2; SD, standard deviation; mRNA, messenger RNA. Data presented as the mean \pm standard deviation.

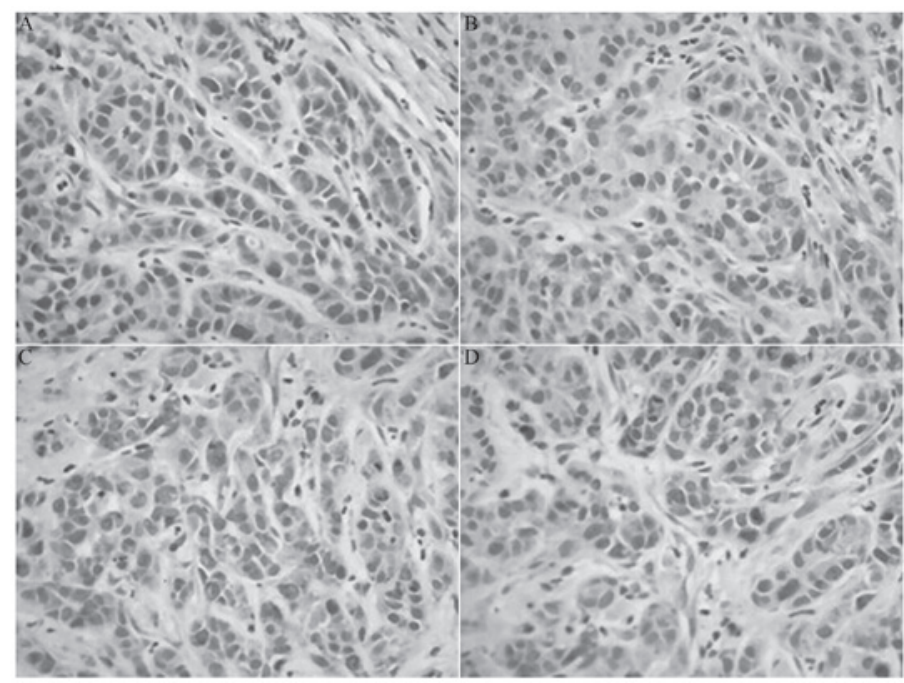

Figure 4. Expression of matrix metalloproteinase-2 protein in each group visualized by immunohistochemistry. (A) Control group, (B) indomethacin group, (C) oxaliplatin group and (D) combination group. Magnification, x200.

obtain. Therefore, the combination of low-dose indomethacin with chemotherapeutic drugs may reduce the significant side effects observed when chemotherapeutic drugs are used alone, meaning that patient tolerance may be improved.

CD44 is a cell adhesion molecule, which primarily mediates cell-cell and cell-matrix adhesion; therefore, it may have a significant role in lymphocyte homing, blood cell generation, migration and metastasis of tumor cells (17). CD44v6 is a CD44 isoform that contains a mutated exon 11, and its expression may alter the composition and functioning of adhesion molecules on the tumor cell surface, enhancing their adhesive abilities (18). This may alter the physiochemical properties of cells, and may contribute to tumor cells obtaining metastatic potential. In the present study, indomethacin treatment alone or in combination with oxaliplatin reduced the expression of CD44v6 protein and mRNA. CD44v6 may be involved in tumor lymphatic metastasis via the following mechanism: Tumor cells that express CD44v6 on the cell membrane may imitate the homing process of activated lymphocytes, transferring from the original tumor to the lymph nodes, and thus obtaining the camouflage of lymphocytes (19). Furthermore, CD44v6 may bind distal lymphatic ligands, meaning that transferred tumor cells are able to escape recognition and killing by the human immune system, and are able to easily enter the lymph nodes to contribute to the formation of metastases (19). CD44v6 is involved in adhesion among cancer cells and basal layer adhesion protein, collagen, fibronectin and type I collagenase (MMP-2 and MMP-9), thus creating conditions for the cancer cells to degrade the ECM (20). As a hyaluronic acid receptor on the T cell surface, CD44v6's NH-2-terminal functional area may connect the hyaluronate of the ECM and basement membrane, anchoring it onto the host's ECM and basement membrane (21), thus regulating cell morphology and movement (22), and laying the foundation for the invasion of tumor cells.

MMP-2 hydrolyzes type IV and V collagen and fibronectin, which are components of the cell basement membrane and ECM, leading to destruction of the basement membrane. Subsequently, this allows tumor cells to more easily break through the ECM-composed barrier structure, and invade surrounding tissues or enter the lymphatic system. Cells are subsequently able to migrate through the lymphatic system, evade immune surveillance, enter distant lymphatic vessels and implant onto the distal lymphatic duct, resulting in the occurrence of distant metastases (23). Furthermore, MMP-2 is able to degrade the vascular basement membrane, causing the production of ECM degradation products, resulting in the production of chemotactic molecules by vascular endothelial 
cells and allowing invasion of vessels (24). In addition, MMP-2 may activate growth factors, regulate cell-cell and cell-matrix adhesion and regulate and promote angiogenesis, therefore promoting the invasion and metastasis of tumor cells (24). In the present study, treatment with NSAIDs alone or in combination with chemotherapeutic drugs reduced the expression of MMP-2 in lung cancer xenografts, indicating that NSAIDs were able to inhibit MMP-2. The potential mechanism through which this was achieved may have been caused by NSAIDs inhibiting the activity and expression of COX-2, thus preventing the activation of secreted MMP-2, and reducing the expression of MMP-2. This would hinder ECM hydrolysis, resulting in a reduction in invasion and metastasis activities. The combination treatment group exhibited significantly reduced MMP-2 expression levels, suggesting that treatment with the two-drug combination led to a synergistic effect on the inhibition of MMP-2 expression.

CD44v6 expression reduces adhesion among tumor cells, and these cells are subsequently prone to leaving the primary lesion (25). CD44v6 also mediates adhesion among tumor cells and the ECM $(6,17)$. MMP-2 degrades the ECM, forming a local dissolution zone, and thus providing an approach towards the metastasis of tumor cells (24). CD44v6 expression activates certain signal transduction pathways in vivo, leading to secretion of proteolytic enzymes and acceleration of ECM degradation (26). Therefore, adhesion and matrix degradation may occur simultaneously during the process of lymph node metastasis, thus having synergistic effects.

Survivin is the strongest apoptosis inhibitor identified to the best of our knowledge, and its tissue distribution demonstrates clear cell selectivity (27). Survivin is not expressed in normal adult differentiated tissues (except for the thymus and placenta), while it is expressed in lung, stomach, colorectal, liver, pancreatic, breast and other types of cancer (28). Survivin is primarily expressed in the G2/M phase of the cell cycle (29), and is the regulation gene of this phase, therefore may be capable of preventing the induction of $\mathrm{G} 2 / \mathrm{M}$ phase apoptosis, and promoting the abnormal proliferation of transformed cells via mitosis. Primarily via inhibition of the activities of apoptosis-regulation terminal effectors (caspase-3 and caspase-7), survivin may be capable of blocking various stimuli that induce apoptosis, thus playing the role of anti-apoptotic agent (30).

p21 may be released from the cyclin-dependent kinase 4-survivin composite body, and bind caspase-3 and inhibit its activity, thus reducing apoptosis (31). Furthermore, survivin may reduce the release of cytochrome $c$, thus demonstrating an anti-apoptotic role. The results of the present study suggested that NSAIDs may reduce the expression of the survivin protein and gene, which may be due to inhibition of the activity of COX-2, and its combination with oxaliplatin demonstrated a synergistic effect towards inhibition of the expression of survivin.

The Wnt signaling pathway is involved in a number of physiological processes in vivo, and its abnormal activation may lead to the occurrence of cancer, fibrosis and other diseases (32). In lung cancer, the Wnt signaling pathway is highly active. Survivin is a downstream target gene of the Wnt signaling pathway, exhibiting dual roles as an inhibitor of apoptosis and promoter of cell proliferation (33). As a downstream target gene of the Wnt signaling pathway, the
Wnt-induced-secreted-protein-1 (WISP-1) is associated with the occurrence of a number of malignancies (34). WISP-1 may promote tumor formation via inhibition of the p53-modulating apoptotic signaling pathway. WISP-1 may induce the expression and secretion of MMP-2, and as MMP-2 may be an intermediary of the WISP-1 response, its effect of degrading the ECM may lead to the consequent movement and metastasis of cancer cells (35). The correlation analysis in the present study revealed that survivin protein expression was positively correlated with MMP-2 protein expression, and was additionally associated with lymph node metastasis, suggesting that NSAIDs may reduce the expression of downstream molecules of the Wnt signaling pathway. This may inhibit the activity of the Wnt signaling pathway, and promote the apoptosis of tumor cells, which ultimately may result in a reduction of tumor invasiveness, as well as inhibition of the occurrence of lymph node metastasis. Combination treatment with indomethacin and oxaliplatin demonstrated good synergy.

In addition, the specificity protein $1(\mathrm{Sp} 1)$ may upregulate the expression of MMP-2 and survivin (36), thus participating in the regulation of proliferation, apoptosis, angiogenesis and other processes in various tumor cells (37). NSAIDs may inhibit the activity of Sp1, thus reducing the expression of survivin and MMP-2; however, the specific mechanism underlying this process remains to be elucidated.

In conclusion, indomethacin treatment alone or in combination with oxaliplatin may significantly inhibit tumor growth inside a lung cancer-nude mouse transplanted tumor model, and is additionally capable of reducing the expression levels of survivin, CD44v6 and MMP-2. The combination of indomethacin and oxaliplatin treatment caused a synergistic antitumor effect via various mechanisms, thus providing potential novel strategies for the treatment of lung cancer.

\section{References}

1. Di Nicolantonio JJ, McCarty MF, Chatterjee S, Lavie CJ and O'Keefe JH: A higher dietary ratio of long-chain omega-3 to total omega- 6 fatty acids for prevention of COX-2-dependent adenocarcinomas. Nutr Cancer 66: 1279-1284, 2014.

2. Han H, Yang S, Lin SG, Xu CS and Han ZH: Effects and mechanism of downregulation of COX-2 expression by RNA interference on proliferation and apoptosis of human breast cancer MCF-7 cells. Mol Med Rep 10: 3092-3098, 2014.

3. Li M, Tan SY and Wang XF: Paeonol exerts an anticancer effect on human colorectal cancer cells through inhibition of $\mathrm{PGE}_{2}$ synthesis and COX-2 expression. Oncol Rep 32: 2845-2853, 2014.

4. Harris RE, Casto BC and Harris ZM: Cyclooxygenase-2 and the inflammogenesis of breast cancer. World J Clin Oncol 5: 677-692, 2014

5. Shao Y, Sun K, Xu W, Li XL, Shen H and Sun WH: Helicobacter pylori infection, gastrin and cyclooxygenase-2 in gastric carcinogenesis. World J Gastroenterol 20: 12860-12873, 2014.

6. Athanassiou-Papaefthymiou M, Shkeir O, Kim D, et al: Evaluation of CD44 variant expression in oral, head and neck squamous cell carcinomas using a triple approach and its clinical significance. Int J Immunopathol Pharmacol 27: 337-349, 2014.

7. Zhao S, He JL, Qiu ZX, Chen NY, Luo Z, Chen BJ and Li WM: Prognostic value of CD44 variant exon 6 expression in non-small cell lung cancer: A meta-analysis. Asian Pac J Cancer Prev 15: 6761-6766, 2014.

8. Hua D, Kong W, Zheng X, et al: Potent tumor targeting drug release system comprising MMP-2 specific peptide fragment with self-assembling characteristics. Drug Des Devel Ther 8: 1839-1849, 2014. 
9. Kamyab-Hesari K, Mohtasham N, Aghazadeh N, Biglarian M Memar B and Kadeh H: The expression of MMP-2 and $\mathrm{Ki}-67$ in head and neck melanoma, and their correlation with clinic-pathologic indices. J Cancer Res Ther 10: 696-700, 2014.

10. Budak M, Korpinar MA, Kalkan T and Tuncel H: Mutation detection in the promoter region of survivin gene on $\mathrm{N}$-methyl-N-nitrosourea induced colon tumor model in experiment. Bratisl Lek Listy 115: 554-556, 2014.

11. Abd El-Hakim TF, El-Shafie MK, Abdou AG, Azmy RM, El-Naidany SS and Badr El-Din MO: Value of urinary survivin as a diagnostic marker in bladder cancer. Anal Quant Cytopathol Histpathol 36: 121-127, 2014.

12. Faibish D, Suzuki M and Bartlett JD: Appropriate real-time PCR reference genes for fluoride treatment studies performed in vitro or in vivo. Arch Oral Biol 62: 33-42, 2016.

13. Turk HM, Camci C, Sevinc A, Bukyukberber S, Sari I and Adli M: Cyclooxygenase-2 expression is not a marker of poor survival in lung cancer. Asian Pac J Cancer Prev 13: 315-318, 2012.

14. Ranger GS: Current concepts in colorectal cancer prevention with cyclooxygenase inhibitors. Anticancer Res 34: 6277-6282, 2014.

15. Quidville V, Segond N, Pidoux E, Cohen R, Jullienne A and Lausson S: Tumor growth inhibition by indomethacin in a mouse model of human medullary thyroid cancer: implication of cyclooxygenases and 15-hydroxyprostaglandin dehydrogenase. Endocrinology 145: 2561-2571, 2004.

16. Di Francesco AM, Ruggiero A and Riccardi R: Cellular and molecular aspects of drugs of the future: Oxaliplatin. Cell Mol Life Sci 59: 1914-1927, 2002.

17. Avoranta ST, Korkeila EA, Syrjänen KJ, Pyrhönen SO and Sundström JT: Lack of CD44 variant 6 expression in rectal cancer invasive front associates with early recurrence. World J Gastroenterol 18: 4549-4556, 2012.

18. Dommann SN, Ziegler T, Dommann-Schener CC, Meyer J, Panizzon R and Burg G: CD44v6 is a marker for systemic spread in cutaneous T-cell lymphomas. A comparative study between nodal and cutaneous lymphomas. J Cutan Pathol 22: 407-412, 1995.

19. Liang YZ, Fang TY, Xu HG and Zhuo ZQ: Expression of CD44v6 and Livin in gastric cancer tissue. Chin Med J (Engl) 125: 3161-3165, 2012

20. Protin U, Schweighoffer T, Jochum W and Hilberg F: CD44-deficient mice develop normally with changes in subpopulations and recirculation of lymphocyte subsets. J Immunol 163 4917-4923, 1999.

21. Matsumura Y and Tarin D: Significance of CD44 gene products for cancer diagnosis and disease evaluation. Lancet 340 1053-1058, 1992

22. Indinnimeo M, Cicchini C, Giarnieri E, Stazi A, Mingazzini PL and Stipa V: Evaluation of CD44 variant 6 expression and clinicopathological factors in pulmonary metastases from colon carcinoma. Oncol Rep 10: 1875-1877, 2003.
23. Röcken M: Early tumor dissemination, but late metastasis: Insights into tumor dormancy. J Clin Invest 120: 1800-1803, 2010.

24. Maehara Y, Kabashima A, Koga T, Tokunaga E, Takeuchi H, Kakeji Y and Sugimachi K: Vascular invasion and potential for tumor angiogenesis and metastasis in gastric carcinoma. Surgery 128: 408-416, 2000.

25. Ko YH, Won HS, Jeon EK, Hong SH, Roh SY, Hong YS, Byun JH, Jung CK and Kang JH: Prognostic significance of CD44s expression in resected non-small cell lung cancer. BMC Cancer 11: 340, 2011.

26. Skubitz AP: Adhesion molecules. Cancer Treat Res 107: 305-329, 2002.

27. Cho HJ, Kim HR, Park YS, Kim YH, Kim DK and Park SI: Prognostic value of survivin expression in stage III non-small cell lung cancer patients treated with platinum-based therapy. Surg Oncol 24: 329-334, 2015.

28. Tao YF, Lu J, Du XJ, Sun LC, Zhao X, Peng L, Cao L, Xiao PF, Pang L, Wu D, et al: Survivin selective inhibitor YM155 induce apoptosis in SK-NEP-1 Wilms tumor cells. BMC Cancer 12: 619, 2012.

29. Andersson SE, Svensson MN, Erlandsson MC, Dehlin M, Andersson KM and Bokarewa MI: Activation of Fms-like tyrosine kinase 3 signaling enhances survivin expression in a mouse model of rheumatoid arthritis. PLoS One 7: e47668, 2012.

30. Jarrin M, Mansergh FC, Boulton ME, Gunhaga L and Wride MA Survivin expression is associated with lens epithelial cell proliferation and fiber cell differentiation. Mol Vis 18: 2758-2769, 2012.

31. Liu W, Zhu F, Jiang Y, Sun D, Yang B and Yan H: siRNA targeting survivin inhibits the growth and enhances the chemosensitivity of hepatocellular carcinoma cells. Oncol Rep 29: 1183-1188, 2013.

32. MacDonald BT, Tamai K and He X: Wnt/beta-catenin signaling: Components, mechanisms, and diseases. Dev Cell 17: 9-26, 2009

33. Bongiovanni L, D'Andrea A, Porcellato I, Ciccarelli A, Malatesta D, Romanucci M, Della Salda L, Mechelli L and Brachelente C: Canine cutaneous melanocytic tumours: significance of $\beta$-catenin and survivin immunohistochemical expression. Vet Dermatol 26: 270-e59, 2015.

34. Katsube K, Sakamoto K, Tamamura Y and Yamaguchi A: Role of CCN, a vertebrate specific gene family, in development. Dev Growth Differ 51: 55-67, 2009.

35. Hou CH, Chiang YC, Fong YC and Tang CH: WISP-1 increases MMP-2 expression and cell motility in human chondrosarcoma cells. Biochem Pharmacol 81: 1286-1295, 2011.

36. Jiang L, Luo RY, Yang J and Cheng YX: Knockdown of survivin contributes to antitumor activity in cisplatin-resistant ovarian cancer cells. Mol Med Rep 7: 425-430, 2013.

37. Mao Y, Chen H, Lin Y, Xu X, Hu Z, Zhu Y, Wu J, Xu X, Zheng X and Xie L: microRNA-330 inhibits cell motility by downregulating Sp1 in prostate cancer cells. Oncol Rep 30: 327-333, 2013. 